\title{
Histobiomechanical Remodeling of the Cervix during Pregnancy: Proposed Framework
}

\author{
Jorge Torres $\mathbb{D}^{\mathbb{D}},{ }^{1}$ Inas Faris $\mathbb{D},{ }^{1,2}$ and Antonio Callejas $\mathbb{D}^{1,2}$ \\ ${ }^{1}$ Department of Structural Mechanics, University of Granada, 18071, Spain \\ ${ }^{2}$ Biosanitary Research Institute, Granada 18016, Spain \\ Correspondence should be addressed to Jorge Torres; geresez@ugr.es
}

Received 14 September 2018; Accepted 12 February 2019; Published 27 February 2019

Guest Editor: Marie Muller

Copyright (C) 2019 Jorge Torres et al. This is an open access article distributed under the Creative Commons Attribution License, which permits unrestricted use, distribution, and reproduction in any medium, provided the original work is properly cited.

\begin{abstract}
Pregnancy involves a gradual change in tissue consistency, where, as gestational age increases a drop in stiffness is noticeable. The extracellular matrix (ECM) of the cervix whose behavior is controlled by the collagen structure is reconfigured, due to hormonal and protein related factors that degraded it. There is an increase in cellular and water content. The collagen maintains a stable percentage, although the synthesis-solubility interaction causes a transformation in the network increasing its diameter and waviness, which destabilizes the mechanical integrity. Further understanding of the remodeling of the cervix becomes a key element in the progress against preterm birth. However, since the woman is in a very delicate state, the available information is scattered. The aim is to provide a framework where the variation of the histological properties is linked with the individual biomechanical evolution of the constituents; therefore a multiscale approach to the problem can be made. The results of different authors were reviewed to obtain values of each component depending on the gestational age, and then they were combined with the mechanical development of each variable obtained from the outputs of numerical simulations of another author. An additive function is proposed, in which the mechanical contribution of each constituent is added to a reference value established for a nonpregnant state. Results show how biochemistry models the mechanical behavior of the tissue through the histology and morphology of the ECM.
\end{abstract}

\section{Introduction}

The anatomical function of the cervix is to serve as a gatekeeper during pregnancy, separating the fetus from the outside. Gestation is a delicate process where there is often lack of information. The possibility of obtaining samples of cervical tissue at any time is solely limited to scientific and at risk cases, which is preventing an adequate breakthrough in the characterization of the etiology of the cervix. This is now an urgent problem in the case of premature birth, the first cause of infant mortality in children under five years of age $[1$, 2]. It remains a major clinical challenge for obstetricians due to the difficulty in its prevention; likewise, some techniques used (such as cerclage and progesterone), while encouraging, present some limitations [3, 4].

It is well known that from the moment a woman gets pregnant biological transformations occur, coming from the combination of the growing pressures exerted by the fetus and biochemical processes, all of which involve the remodeling of the cervical tissue. This implies changes in the morphology of the tissue to the point of altering mechanical properties [5]. The cervical stroma, which is the part that has a connective role, has a composition that consists of a collection macromolecules necessary for pregnancy and to produce muscle functions [6]. Additionally, there is a set of molecules embedded in an extracellular matrix (ECM), which provides structural support to the cells. Collagen fibers are its main constituent, which form a cross-linked network intertwined with the protein elastin, with both being surrounded by a viscous substance of glycosaminoglycans (GAGs) and water [7]. Collagen provides tensile strength and firmness through its hierarchical composition, made of small fibers comprising a bundle [8]. Furthermore, its waviness confers anisotropy and heterogeneity to the tissue. With these components, a viscoelastic behavior is exhibited, with great deformations and relaxations. In low stress elastin acts as a spring returning quickly to its state [9]. GAGs, due to their viscous character, under compression serve as dampers [10]. The water modifies 
TABLE 1: Stroma evolution during gestation. Percentages refer to dry tissue, except for water and dry tissue, which are in relation to the total.

\begin{tabular}{|c|c|c|c|c|c|}
\hline \multicolumn{6}{|c|}{ Content (\%) } \\
\hline Constituents & Nonpregnant & Early & Late & Postpartum & Reference \\
\hline Water & 78 & 75 & 83 & 76 & [35] \\
\hline Dry tissue & 22 & 25 & 17 & 24 & - \\
\hline Collagen content & 71 & - & 78 & - & [11] \\
\hline Collagen solubility & 17 & 75 & 76 & 88 & {$[34]$} \\
\hline Elastin & 1.33 & - & 0.73 & - & {$[51]$} \\
\hline GAGs & 1.6 & - & 2.6 & 1.2 & {$[24]$} \\
\hline Cells & 8 & - & 15 & - & [6] \\
\hline
\end{tabular}

the mechanical relations in the cervix entering a state of unbalance, disorganizing the components [11]. Therefore, the ECM ensures the integrity of the tissue during gestation.

The remodeling process is a progressive transformation of the appearance of the tissue that is translated into four phases which are superimposed as they occur until birth, namely, softening, ripening, dilation, and postpartum [12]. There exist a series of factors, mainly hormonal and protein related, that promote an inflammatory state [13] that degrades the ECM and enhance tissue distensibility. The mechanical response during gestation is a decrease in stiffness, which is governed by the structure of collagen. There is a reduction of crosslinking between fibers; besides, the distinctive arrangement of the fibers changes, from being aligned to curling [14].

As can be inferred from the above, the morphology of the cervix during pregnancy is a complicated subject to study, where the greater information collected comes from the moments before and after delivery. Experimental data of the morphological and biochemical characteristics at the microscopic level is essential to monitor trends and to be able to program specific actions since the mechanical properties at the macroscopic level of the tissue are related to them $[5,15]$. Notwithstanding, the complex structure of the cervix hinders the analysis of individual changes in each component, whose behavior is interrelated.

Hence, the main objective of this work is to provide a first approach in a temporal prediction of the stiffness evolution of the cervix, where the contribution of each constituent will be highlighted. To get here, an histobiomechanical framework that materializes in a curve of stiffness versus gestational age is built. Besides, the relationships established between biochemistry and histology will be shown.

\section{Materials and Methods}

Histological transformations in the tissue are responsible for variations in consistency. As a starting point, the basis of this study is on the multiscale approach proposed by Peralta [16], which indicates the interconnections between components, which allows filling gaps in knowledge. In order to study the mechanisms that govern internal processes of the remodeling, a three-step procedure is proposed: (1) to fit curves for the chosen values of the content of the fundamental constituents of the cervical stroma derived from the literature, (2) to adapt the results of Peralta et al. [15] where they computed a numerical relationship between histological and morphological changes and the tissue elasticity, and (3) to combine the previous results. It is suggested that the process is inverted, beginning from the mechanical contribution of each independent element to end with a real evolution of the whole set.

2.1. Content of the Components and Fitting. Since each person is different, whether by obstetrical or medical history, the values chosen to represent each component should be representative of the important variations recorded. Selection criteria are proposed based on existing information: (i) It must follow the behavior recorded by most studies. Some measures deviate much from the general trend and remain isolated as cases without validation by other sources. (ii) The biochemical analysis that has been used for measuring is crucial. First of all, the result should be in dry weight, because large variations are registered when working with wet weight [17]. Secondly, the assay should be standardized to contrast it with other sources. (iii) Sufficient data should be available. Many studies have evaluated the content of some components only at one point in pregnancy, usually at the beginning, so that evolution of these cannot be envisaged.

Selected data are summarized in Table 1 . The gestation age corresponds to 0 weeks in nonpregnant, 12 weeks in early, 38 weeks in late, and 42 weeks in postpartum phase.

The chosen values are fitted to time evolution curves with the intent to relate the gestational age to absolute values (\%) of each ECM component. Many different mathematical models for growth can be useful [18], which can vary in flexibility and number of parameters needed. For example, the development of many organisms is represented by logistic curves [19] while cell growth is represented with exponential models [20]. In these situations, nonlinear modeling stands as a tool able to estimate characteristic parameters, which could establish a more reliable link between gestational age and component content. By convention, a balance between variance and bias is always sought. However, due to the scarcity of data that has been mentioned, a good fit is desired, prioritizing the bias and discarding the variance. The curves can be fitted to the data using nonlinear least squares. This procedure is conducted by a Levenberg-Marquardt algorithm [21].

The following content describes the temporal evolution of each ECM component and its characteristics.

2.1.1. Collagen. In terms of dry weight, the collagen content remains practically constant. This has not been measured 
by many researchers, as it is a rigorous process. It is fitted to a logistic curve (see (1)) that simulates how the increase of collagen at first is important to then stagnate due to the interaction solubility-synthesis that will be discussed later.

$$
\frac{d C}{d t}=C(k-\delta)
$$

where $C$ is the collagen content in function of the time, $k$ is the growth rate, and $\delta$ is the steepness of the inflection zone, related to the rate of synthesis of new collagen.

It is expected that as collagen turnover increases, a synthesis-degradation process will be appreciated, affecting the morphology [22] and reducing the mechanical strength. The cervical fibroblasts secrete tropocollagen that self-assembles into fibrils, and then they aggregate to form a collagen fiber [23]. It is believed that as solubility increases (on account of proteases, collagenases, lysyl oxidase, etc.), the synthesis process is repeated more frequently, which means that by not having enough time to be properly disposed of, the collagen is created in new directions and increases disorganization [24]. These newly synthesized fibrils are combined with each other, losing their combined strength due to weaker cross-links, thus favoring degradation [25]. In short, the faster this synthesis is, the greater solubility will be observed, becoming a feedback process.

A crystallization-like system is proposed for synthesis representation. The formation of a new crystal starts with a nucleation process [26], a thermodynamical phase that is dependent on the solute concentration. Heterogeneous primary nucleation occurs when there is an increase in crystal synthesis due to interaction with other particles, which catalyzes this process by providing sustenance.

$$
\frac{d S}{d t}=L+k \cdot t^{n}
$$

Equation (2) is expressed in terms of tissue remodeling, with $S$ being the synthesis rate, $L$ the value at time $0, k$ the growth rate, and $n$ an empirical exponent from crystallization trials.

As discussed above, the interaction solubility-synthesis is key to understand the loss of tensile strength, since the concentration of collagen does not seem to vary significantly and therefore could not be the cause of the changes. This is perceived as a nutritional intake, where the created collagen is destroyed, but when synthesis exceeds degradation, a saturation environment is reached. To represent this, a MorganMercer-Flodin sigmoidal model is suggested (see (3)).

$$
S_{o}=\gamma-\frac{\gamma-\alpha}{1+(k t)^{\delta}}
$$

where So is the solubility rate, $\gamma$ is the saturation value, $\alpha$ is the initial nonpregnant value, $k$ is the growth rate, and $\delta$ is the point where degradation exceeds collagen creation.

Following the synthetic model created by Peralta et al. [15] (outlined in the following section), the morphology of the collagen fibers can be described using three parameters: diameter, interfibrillar space, and waviness. The range of variation in diameter and interfibrillar space increases with gestation $[27,28]$, and thus it is expected to evolve in a linear fashion (see (4)):

$$
\frac{d D}{d t}=k
$$

with $D$ being the value over time and $k$ the slope of the function (the evolution ratio).

Pinheiro et al. [29] examined the evolution of collagen waviness in the pubic joint of pregnant mice, which resembles the behavior of connective tissues in humans. They measure the crimp angle and length directly on the electron micrograph confirming a decrease in angle and consequently an increase in length. The fibers were arrays of parallel fibrils relatively straight at the beginning. As gestation progressed, the fibers were thicker due to the deposition that packed them together, following a wavy course. When the tensile forces become palpable near delivery, this poorly bonded set of fibers tends to easily stretch and separate, leaving fibrils alone, paving the way to complete remodeling. It is hypothesized that, given that it is a fast process, the fibrils do not have time to bond properly which is causing increasing disorganization. By means of the model of Cacho et al. [30] a beta distribution of the crimp is assumed. They generate a data set randomly distributed within a range in the $\mathrm{X}$ axis, so that each associated point on the $\mathrm{Y}$ and $\mathrm{Z}$ axes was distributed with a zero mean normal. With this conditioning, the data are considered as Gaussian white noise, so that its probability density function (PDF) is only characterized by the variance $\sigma^{2}$.

Three distributions are conceived, ranging according to the phases of early, mid, and late pregnancy. Equation (5) presents the distribution and its parameters:

$$
\begin{aligned}
\beta(a, b) & =\frac{x^{a-1}(x-1)^{b-1}}{B(a, b)} \\
B(a, b) & =\frac{\Gamma(a) \Gamma(b)}{\Gamma(a+b)} \\
a & =\left(\frac{1-\mu}{\sigma^{2}}-\frac{1}{\mu}\right) \mu^{2} \\
b & =a\left(\frac{1}{\mu}-1\right)
\end{aligned}
$$

where $B()$ is the beta function, a normalization constant to ensure that the total probability integrates to 1 , and $\Gamma()$ is the gamma function. The shape parameters of the distribution $(a, b)$ configure its scale and position. This output will give references about the fitting parameters and the behavior of the waviness.

Taking into account the interaction solubility-synthesis and the values of the probability distribution, a similar evolution is anticipated. This is reflected in a logistical function (see (6)).

$$
\frac{d W}{d t}=W(k-\delta)
$$


where $W(t)$ is the collagen waviness in function of the time, $k$ is the growth rate, and $\delta$ is the steepness of the inflection zone, where solubility overcomes synthesis.

2.1.2. Water. The interstitial fluid controls the stiffness response of the tissue. It presents a similar development to hyaluronic acid (HA) that affects collagen organization [31]. When the content increases before delivery, about $5 \%$, it enters a state of nonequilibrium, which reduces the elasticity. As can be observed in Table 1, hydration follows a cyclic behavior represented in

$$
\frac{d^{2} H(t)}{d t^{2}}+H(t)=0
$$

where $H(t)$ is the hydration content in function of time.

2.1.3. Cells. In general terms, there is an increase in vascularization. In the nonpregnant state, there are approximately 8 to $10 \%$ of fibroblast, epithelial, and smooth muscle cells. Near delivery there is an enormous increment of cells due to the proinflammatory state [10]; as a result, an exponential growth rate characterizes the phenomena (see (8)), although at some point in the postpartum this will be limited by the return of the nonpregnant state.

$$
A\left(\frac{d C}{d t}-k C\right)=0
$$

where $C(t)$ is the content of the cell in function of time, $A$ the initial value, and $k$ the growth rate.

2.1.4. GAGs. As a whole, they show a very rapid and abrupt evolution when it comes to labor, which can be divided into two phases and then fitted to an exponential model; see

$$
G(t)=a e^{b t}+c e^{d t}
$$

where $G(t)$ is the GAGs content in function of time, $a$ and $c$ are the initial values, and $b$ and $d$ are the growth rates.

2.2. Contribution to the Stiffness of the Constituents. This section summarizes the results of the work of Peralta et al. [15]. The greatest difficulty in this study came from the individual description of each component. To tackle this, a numerical model that relates the architectural changes in the microscale to the propagation of shear waves was proposed. This made it possible to obtain quantitative information, in elastic terms of the shear modulus.

The effect of each constituent was studied independently, i.e., not affecting the behavior of the whole; when a content varied, reference values were set. The reference configuration for the model (see Table 2) was established at typical values of the nonpregnant state; for that reason any change in content over time impacted on the computed mechanical properties.

They proposed different models of analysis, and here the one that has the fibers arranged longitudinally and perpendicular to the propagation is adapted. The election is based on a recent study by [32], where pregnant samples were analyzed to find that the cervix section is mostly composed
TABLE 2: Reference input values of the model. Source: [15].

\begin{tabular}{lc}
\hline Fiber diameter & $5 \mu \mathrm{m}$ \\
Interfibrillar space & $1 \mu \mathrm{m}$ \\
Fiber waviness (sd) & $15 \mu \mathrm{m}$ \\
Fiber fraction & $20 \%$ \\
Hydration & $80 \%$ \\
GAGs & $1.5 \%$ \\
Cells & $8 \%$ \\
\hline
\end{tabular}

of circumferential collagen, which is the direction that will determine the behavior before dilation. The rest is exhibited in a radial form. Nevertheless, this implies that if only the effect of one profile is taken into account, erroneous conclusions can be reached, because waviness configures the network in a random way. So it is proposed to implement a probability density based on directionality within a circle, similar to a normal distribution. This method will be adopted for obtaining the reference value of the stiffness of the cervical tissue and for the final contribution of the waviness. It is estimated that the greatest contribution of waviness comes from the perpendicular profile; however, the parallel profile helps in setting up the network due to the crimping of the fibers. The contribution of this profile is calculated with a Von Mises distribution (10); its density function for the angle $x$ is

$$
f(x)=G_{n} \frac{x}{\pi / 2}+G_{p} \frac{\pi / 2-2}{\pi / 2} \frac{e^{k \cdot \cos (x-\mu)}}{2 \pi I_{0}(k)}
$$

with $G_{n}$ and $G_{p}$ being the elastic contribution of the perpendicular and parallel profile, respectively, $\mu$ where the distribution is clustered, $k$ a dispersion measure that concentrates the points in $\mu$, and $I_{0}$ the modified Bessel function of order 0 . Following this, the cumulative density function (11) is computed in the interval $0-\pi / 2$.

$$
F(x)=\int_{0}^{\pi / 2} f(x) d x
$$

Regarding the behavior of the components, several differences between this work and Table 1 can be found. The concentration of collagen is progressively reduced in the numerical model, which became effective by decreasing its mechanical contribution; however, there is a slight increase. This decision does not affect this work, as the stress and the constant synthesis of immature fibers underwent by the collagen network are causing the loss of tensile strength. On the other hand, GAGs content has been informed to increase until labor and then quickly drop. Nonetheless, the synthetic model considered that GAGs decrease for the beginning, which means that fewer particles were formed between collagen and less disorganization can be appreciated. For this reason, it is considered that there is no effective change in the consistency by this component.

The final results are fitted with polynomial functions in order to get an interpolation that collects all the values from the range. The most relevant properties of the model considered are fiber diameter, fiber waviness, hydration, 
and cells. Since interfibrillar space and diameter are linked characteristics, they have a joint contribution represented as a single variable. Although GAGs have been found to have no appreciable effect on stiffness, they are known to alter the mechanical structure of the tissue $[10,33]$. This is probably due to the fact that GAGs affect other components, mainly collagen, so that its action is indirect, and therefore its independent variation in concentration could not quantify a physical interaction with other elements.

2.3. Gestational Age against Cervical Stiffness. The last step is to combine these two previous results to create a curve that relates gestational age to cervix stiffness. Growth curves (gestational age dependent variables) are entered into the outcome of numerical simulations (mechanical development of each variable). Once implemented, each component will contribute to the consistency, either by adding to or subtracting from the reference value set for the nonpregnant state; therefore it works in relative terms. The influence on the reference value can be represented as an analogy of a Taylor series, as in (12).

$$
\begin{aligned}
G(D, W, H, C)= & G(r e f)+\Delta G(D i a)+\Delta G(\text { Wav }) \\
& +\Delta G(\text { Wat })+\Delta G(C e l)
\end{aligned}
$$

$G(D, W, H, C)$ represents the final contribution of the elements considered, $G(r e f)$ is the reference value, and $\Delta G(D i a)$, $\Delta G($ Wav $), \Delta G($ Wat $)$, and $\Delta G(\mathrm{Cel})$ are the relative inputs of the diameter and waviness of the collagen, water, and cells, respectively.

\section{Results and Discussion}

First of all, the chosen data will be examined for each component; readers are referred to Table 1 . Next, the solution of the differential equations and the parameters obtained in the nonlinear fittings and curves will be shown. Second, the stiffness curves of the synthetic model of each variable are adopted through polynomial fittings and the reference stiffness value is obtained. Finally, the relative contribution of each component is taken and a complete model of the cervical mechanical evolution is provided.

The latest data recorded for the collagen content was made by Myers et al. [11], where there is a small increase in collagen content, reflected in (13). Results are displayed in Figure 1.

$$
C(t)=\alpha+\frac{\gamma-\alpha}{1+e^{(k-t) / \delta}}
$$

In the first few weeks the rate of solubility shows a very large increase, and then it stabilizes and stagnates near the end (see Figure 2). This behavior can be modeled with the data registered by Uldbjerg et al. [34].

The interaction solubility-synthesis configures the ratio of synthesis. There must be a balance to preserve the structural integrity, and so, synthesis is opposed to solubility action with similar values (see Figure 3 ).

The data for the diameter and interfibrillar space were taken from the model of Peralta et al. [15]. As the other components of the ECM develop, they will affect the morphology

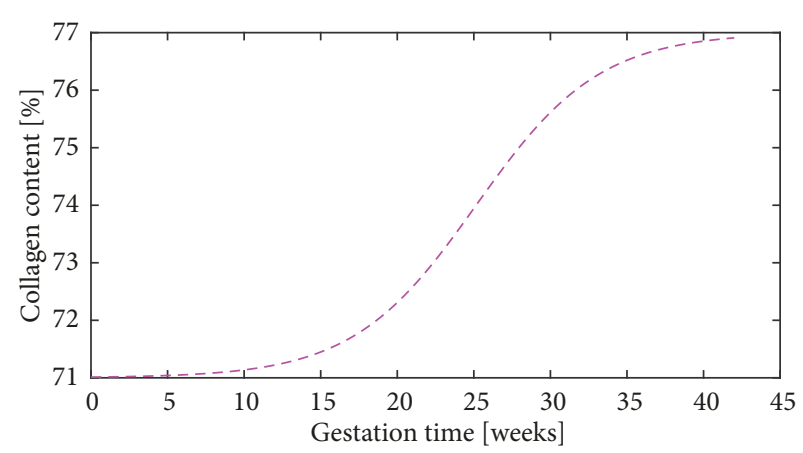

FIGURE 1: Collagen content evolution during pregnancy, with $\alpha$ being the initial value and $\gamma$ the maximum content. Parameters: $k$ $=25.15, \delta=4.035, \alpha=71$, and $\gamma=77$.

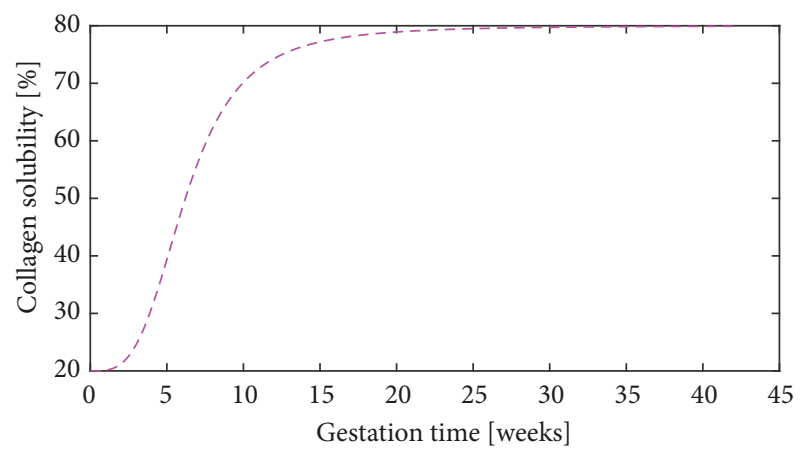

FIgure 2: Collagen solubility evolution during pregnancy. Parameters: $k=0.161, \delta=3.42, \alpha=20$, and $\gamma=80$.

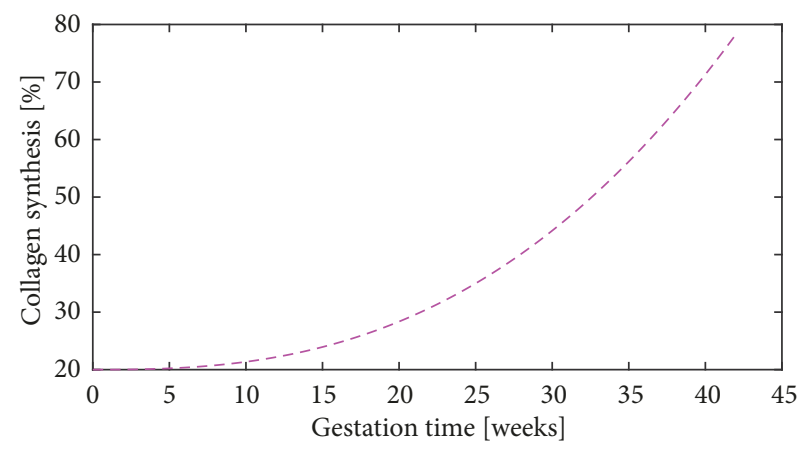

Figure 3: Collagen synthesis evolution during pregnancy. Parameters: $L=20, k=0.003281, n=2.618$.

of the collagen; in this case, it is assumed in a linear fashion (see Figure 4). The range for the diameter is established in (5, 10) $\mu \mathrm{m}$ and for the interspace in $(1,4) \mu \mathrm{m}$. The models for diameter and space are illustrated in (14), respectively.

$$
\begin{aligned}
F_{d} & =0.119 t+5 \\
I_{d} & =0.0714 t+1
\end{aligned}
$$

In the case of waviness, the fibers increase its crimping in agreement with the synthesis of collagen. The range of the variance $\sigma^{2}$ for the beta distribution has been restricted to 5 $45 \mu \mathrm{m}$. It has been quantified from the fiber fraction with 


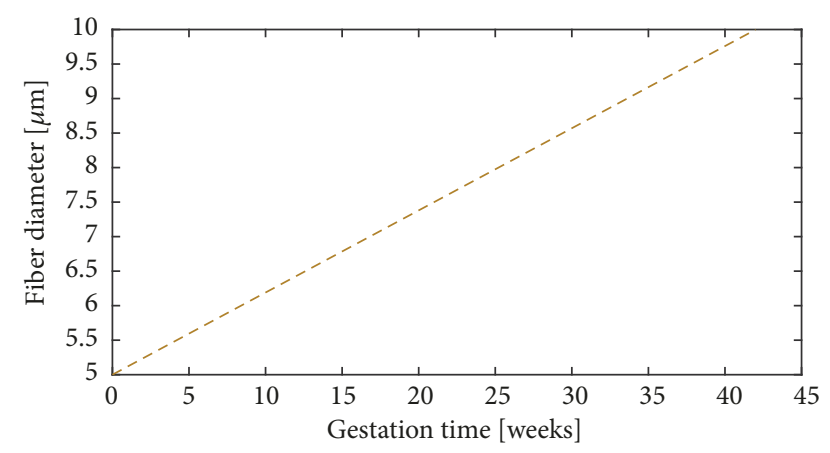

FIGURE 4: Collagen diameter evolution during pregnancy.

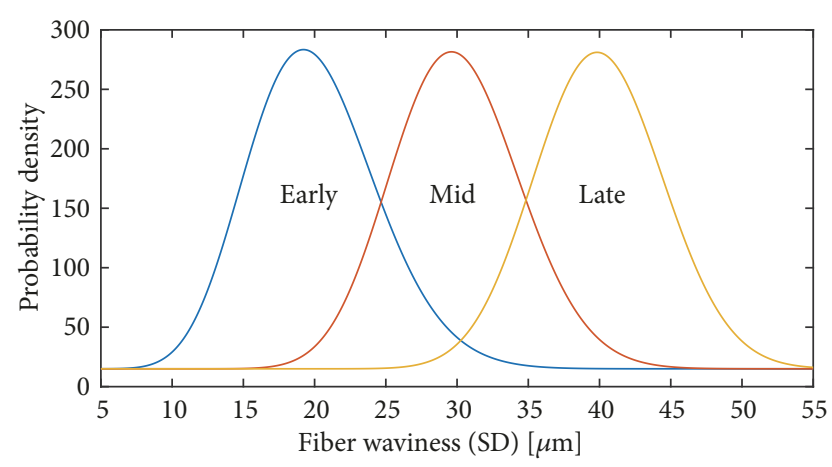

Figure 5: Waviness probability density function. Parameters: for early $(a=15.8 ; b=63.2)$, for mid $(a=31.2 ; b=72.8)$, and for late phase $(a=47.6 ; b=71.4)$.

respect to the total ECM and the morphology observed in microscope images [16]. Since the $\beta$ distribution is restricted to (0-1), it is possible to shift and scale the output through (15).

$$
5+(45-5) \cdot \beta(p d f)
$$

The shape parameters of the distribution are computed according to the phase of pregnancy, where it is assumed that the mean $(\mu)$ of the early phase is $15 \mu \mathrm{m}$, for the mid phase $25 \mu \mathrm{m}$, and for the late phase $35 \mu \mathrm{m}$, with a variance $\left(\sigma^{2}\right)$ for all of $0.002 \mu \mathrm{m}^{2}$. These values once calculated are presented in Figure 5, along with their probability density. The results allow estimating key evolutionary points in the waviness. There is a minimum around $8 \mu \mathrm{m}$ and a maximum around 55 $\mu \mathrm{m}$. In early phase the most probable value is approximately $20 \mu \mathrm{m}$, in mid phase is $30 \mu \mathrm{m}$, and in late phase is $40 \mu \mathrm{m}$.

Due to their reliance on collagen synthesis, a similar evolution is expected for waviness, starting with cautious crimping and then undertaking a remarkable increase, reaching a value that could not be higher. Equation (16) is the logistic function solution. Figure 6 represents the estimated curve with its parameters.

$$
W(t)=\alpha+\frac{\gamma-\alpha}{1+e^{(k-t) / \delta}}
$$

Some authors have measured the water content in the cervix, to conclude that there is an increase of $5 \%$. However, only a few were able to complete the data in all the stages

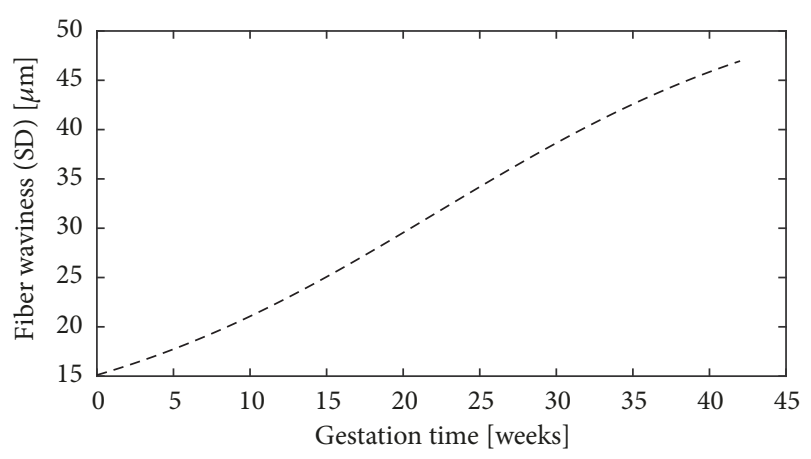

FIGURE 6: Fiber waviness evolution during pregnancy. $\alpha$ is the lower crimping value and $\gamma$ the maximum waviness reached. Parameters: $k=22.26, \delta=12.42, \alpha=8.5$, and $\gamma=54.8$.

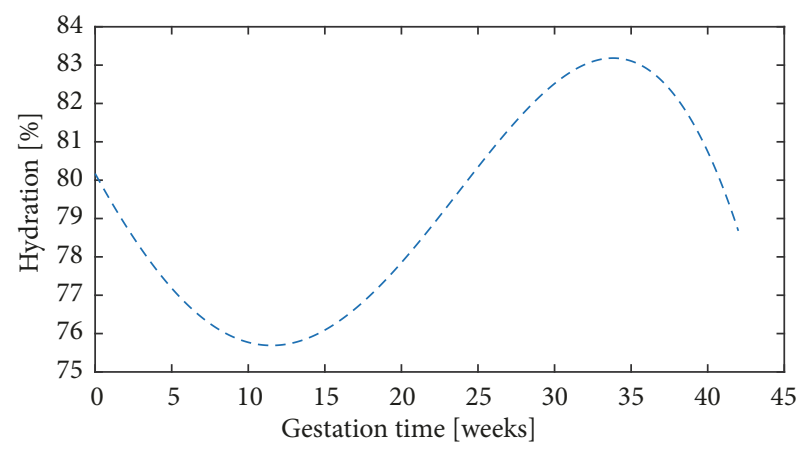

FIGURE 7: Water content evolution during pregnancy. Parameters: $a_{1}=712.3 ; a_{2}=636.7 ; b_{1}=0.05475 ; b_{2}=0.0587 ; c_{1}=0.705 ; c_{2}=3.788$.

of pregnancy. Anderson et al. [35] obtained a sinusoidal relationship, that is translated into (17), where $a_{i}$ is the amplitude of the change, $b_{i}$ the frequency according to the stage of pregnancy, and $c_{i}$ the phase that indicates the initial percentage. In Figure 7 the transformation is visualized.

$$
H(t)=a_{1} \sin \left(b_{1} t+c_{1}\right)+a_{2} \sin \left(b_{2} t+c_{2}\right)
$$

The cell proliferation is based on the protection and preparation for the last phase of pregnancy, where there are a number of chain reactions that affect the hormones responsible for pregnancy. Due to this, an exponential evolution (see (18)) is presented, via the data of Oxlund et al. [6]. Figure 8 shows the output.

$$
C(t)=A e^{k t}
$$

The last constituent studied is GAGs. They have a strong relationship with collagen morphology and water content. Therefore, they begin with gradual growth, to reach birth and fall exponentially (see Figure 9). The data of Shimizu et al. [24] is faithful to this description.

In the next point, the reference mechanical value of the cervix has been calculated. It comes from the conjunction of the profiles of the numerical model and the distribution of Von Mises. The module $\mathrm{G}$ for the perpendicular profile is $14 \mathrm{kPa}$ and for the parallel is $166 \mathrm{kPa}$. The distribution is clustered in $\mu=\pi / 2$ and $\mathrm{k}=2$. The computed value after using the cumulative distribution function is $31 \mathrm{kPa}$. 


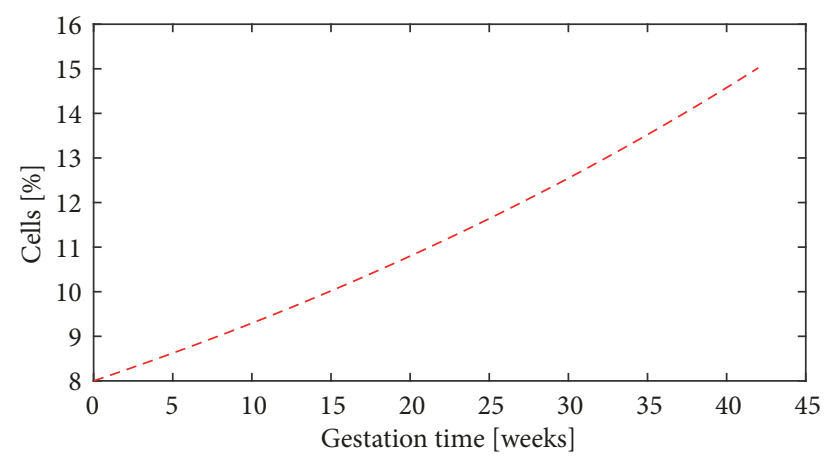

Figure 8: Cells content evolution during pregnancy. Parameters: $\mathrm{A}=8 ; k=0.015$.

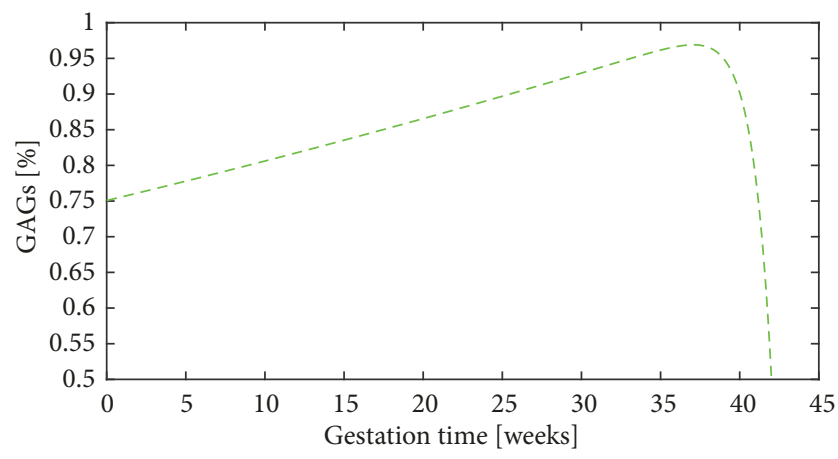

FIGURE 9: GAGs content evolution during pregnancy. Parameters: $a=-3.3 \cdot 10^{-16} ; b=0.8326 ; c=0.7507 ; d=0.007117$.

The response of the diameter and waviness of collagen, water, and cells, which are the elements considered for the final model, are adapted with polynomial fittings. Figure 10 displays the adaptation.

The combination of both results, introducing the temporal evolution in the mechanical contribution and subtracting the initial value given to each constituent, is presented in Figure 11. It is emphasized that the collagen morphology is mainly responsible for decay in stiffness. Hydration has no appreciable effect due to its scale a priori, while cells increase their production and counteract the loss of distensibility caused by collagen.

The final model is displayed in Figure 12. The central value is $31 \mathrm{kPa}$, and then terms of the relative contribution of each component are added to simulate a complete function. At first, the drop in stiffness is noticeable. By week 25, the synthesis-solubility interaction of collagen stabilizes and this is when cell recruitment becomes palpable, balancing the mechanical integrity of cervical tissue.

Several authors have conducted feasibility studies regarding the use of shear wave elastography on pregnant women. They are based on the SSI technique [36] that is able to quantify the shear wave speed (SWS). Once this speed $\left(\mathrm{C}_{\mathrm{s}}\right)$ is computed and under the assumption of a purely elastic and incompressible medium the shear modulus $(\mu)$ can be described as $\mu=\rho \mathrm{C}_{\mathrm{s}}^{2}$, with $\rho$ being the density of the tissue. Bearing this in mind, the range of values stemming from this final model are consistent with the study of Peralta et al. [37]. On the other hand, in the works of Muller et al. and Hernandez-Andrade et al. [38, 39], although they obtained a narrower and smaller value range, they observed a similar trend; from the first weeks there is a notable decrease in the stiffness that in mid pregnancy softens. For its part, in a recent study of Carlson et al. [40] using a prototype of shear waves, they noted that stiffness is reduced by half from the first to the third trimester, with values close to those of this work.

Furthermore, this elastic transformation of the cervix can be assimilated with the remodeling that is experienced in the different phases of pregnancy.

Cervical softening is the first and longest phase that commonly starts in the first month. At this moment it begins a slowly increasing turnover of ECM components, which shows a decline in resistance, associated with unordered collagen fibers, elastic tension, increased water content, and proteoglycans [41]. In the nonpregnant cervix the epithelial cells are scant; however, during pregnancy the hormone relaxin is implicated in their proliferation when the synthesis of some prostaglandins (PGs) begins to be noticed and the cytokine interleukin-8 (IL-8) appears [42]. From this moment the cells are configured as the main support to the structural integrity. They secrete mucus plug [43], a physical barrier against infections, as well as a proliferation of fibroblasts. Yet, due to regulation in the ECM, the collagen fibers begin to lose strength in their state of creation and assembly, where the matricellular proteins thrombospondin 2 (THBS2) and tenascin $\mathrm{C}(\mathrm{TnC})$ modify the collagen architecture [28]. This is reflected in the first 25 weeks of the graph.

Cervical ripening begins a few weeks before delivery and is characterized by rapid changes in the cervix, where integrity is being lost in order to prepare for the delivery. There is a marked increase in the synthesis of proteoglycans, collagen, and hyaluronic acid (HA), a hydrophilic glycosaminoglycan, which interacts with epithelium cells and proteoglycans as versican. HA is in a large molecular weight form that has been said to favor tissue hydration, resulting in a viscoelastic material, which unbalances the structure of the collagen network, dispersing and reducing its mechanical resistance due to increasing collagen solubility $[44,45]$. The cervical epithelium continues to provide a mucosal barrier against infection while regulating steroid hormones to provide a rich estrogen environment. In order to initiate this phase, it is necessary that the activity of progesterone decays, since it has been proven that introducing its inhibitors during softening cause it to reach maturation sooner [46], proving that progesterone is a factor that maintains pregnancy. The loss of this hormone helps the estrogen to reach its receptors, facilitating the activation of ripening.

At term there must be a dilation of the cervix, which is preceded by biochemical changes caused by a new hormonal regulation, contractions of the myometrium, and the tension of the fetal protrusion, triggering the maximal loss of tensile strength. This process involves the infiltration of leukocytes, PGs, proteases, and collagenases into the ECM [13]. The protective mucus plug is expelled; in addition, it comes to a phase of effacement where the cervix narrows its walls and shortens, to reach $10 \mathrm{~cm}$ in diameter. The activity of 

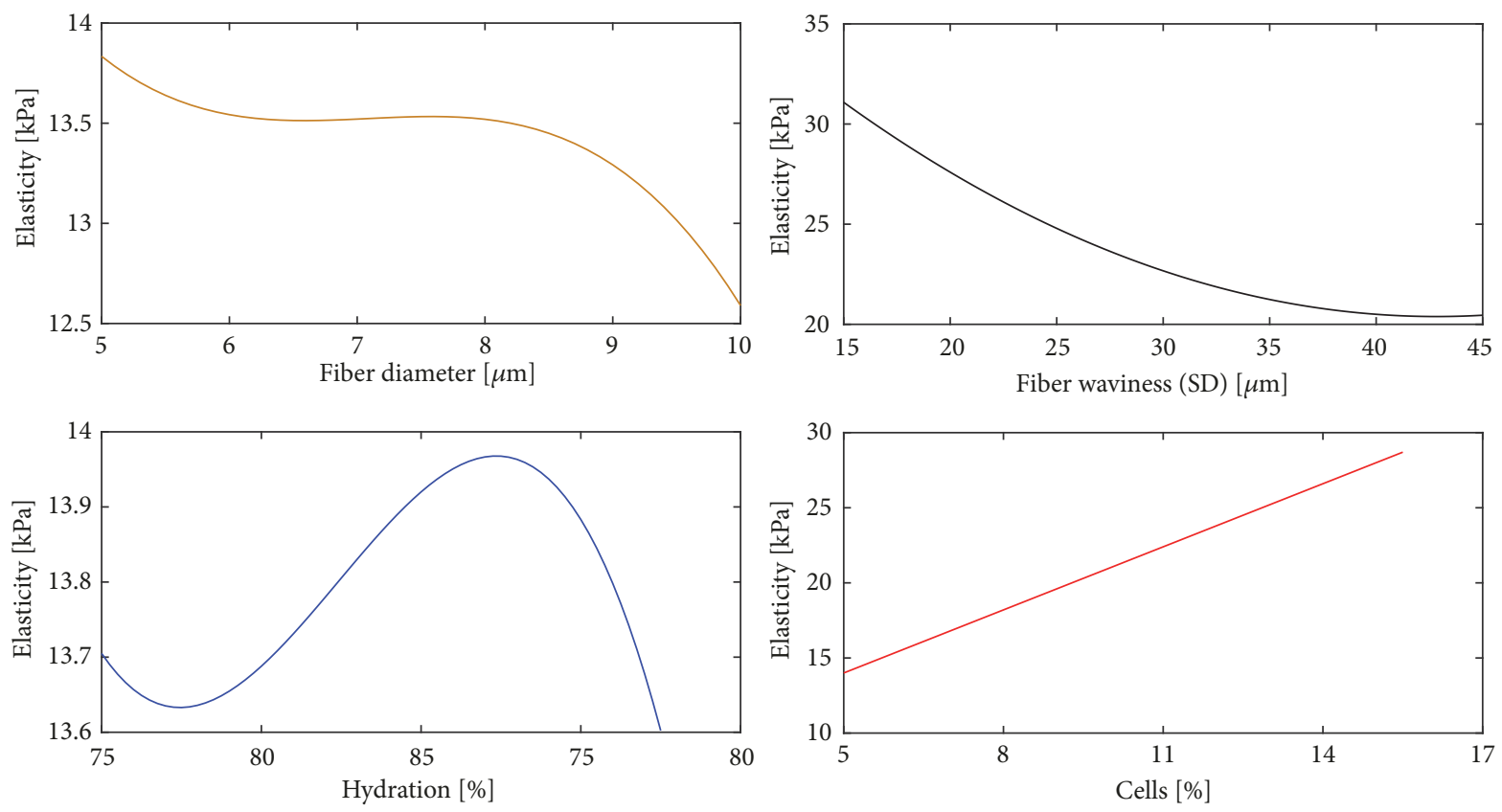

Figure 10: Content of each component versus elasticity. Polynomials of degree three were used for diameter and hydration, degree two for waviness, and degree one for cells.
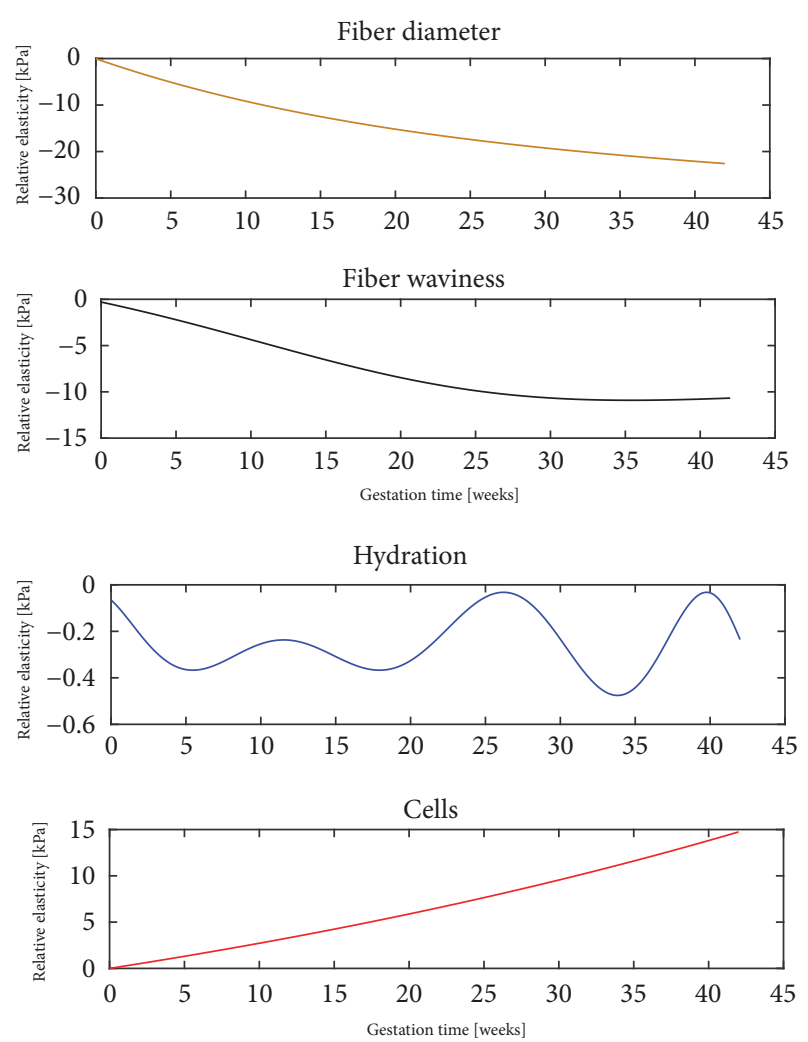

FIGURE 11: Content of each component versus elasticity. The polynomial degree for diameter and hydration is three, for waviness is two, and for cell is one.

the enzyme hyaluronidase is increased, breaking down HA bonds and promoting a shorter duration of labor by cause of loss of cervical mechanical strength [47]. It has been

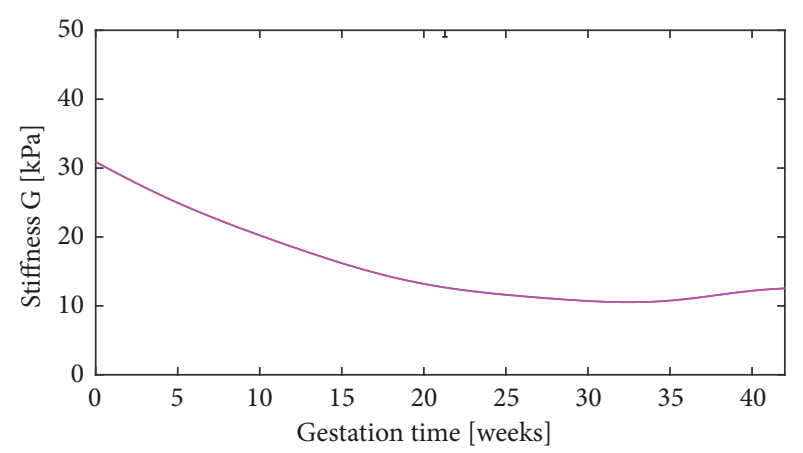

FIGURE 12: Evolutive biomechanical model of the cervix.

proven that PGs have a crucial function in the late term. The application of exogenous PGs increased collagen solubility and altered ECM components, imitating cervical ripening and dilation effects. Notwithstanding, the prostaglandins PGE2 and PGF2 do not increase its content which has left a gap as to its operation, even introducing a progesterone inhibitor induced ripening, but no rise in PGs was registered $[48,49]$. To complete pregnancy it is necessary that the female reproductive system is transformed from a static formation to an active one with the coordination of its components. This coordination will lead to the final contractions that are transmitted through gap junctions. The fetus intervenes through the placenta, which creates different hormones. This is reflected in changes in progesterone-estrogen ratio, upregulation of oxytocin, PGs, and the decrease of nitric oxide (NO). The previous phases (ripening and dilation) can be grouped from week 25 to week 38 . The stiffness drop is no longer so marked. On the one hand, collagen continues to lose its structure, but on the other hand, cells 
together with hormonal activity mean the infiltration of many components.

The postpartum phase, the uterine involution, is meant to recover the tensile strength of the tissue, to avoid environmental contamination and to prepare for ensuing pregnancies. An increase in leukocyte cells allows the renewal of tissue functionality, breaking down components causing weakness and disorganization of the ECM through the secretion of the enzyme protease. On the other side, there is an upregulation of synthesis of ECM constituents to return to a nonpregnant state [50] and loss of tissue hydration. The last stretch of the plot is remarkable for being the only one in which stiffness begins to rise.

This flow explains and validates decisions on both the content of components and their evolution.

This work has certain limitations. The resulting curve was achieved through values from a simulation, which does not necessarily have to be correct. With real values, the curve would be more reliable and optimized. The fit models have been chosen for convenience to simulate the behavior explained, so that some parameters are not clearly defined. The advantage of studying the components individually becomes a constraint as soft tissue remodeling is usually approached from the perspective of mixing theory. There is a mass balance where the constituents make a continuous exchange of percentages of properties. In the work of Myers et al. [52] it is proposed to adopt the tissue composition as state variables that mathematically describe the state of a dynamic system. In the future, this model can be used to build a continuum biomechanical framework.

\section{Conclusions}

A framework of histobiomechanical properties of the cervical stroma is proposed. A literature review was carried out to explore the contributions of several authors, and then nonordinary developments have been identified. In the case of hydration there is a cyclical tendency, probably related to the feeding of the fetus and eventually when water breaks. GAGs decrease exponentially when delivery arrives. It may be thought that it is due to the increase in mucus plug that somewhat dissolves the polysaccharides of GAGs, allowing a hyperstretching of cervical tissue by removing most of its viscosity. The rest of the constituents follow an increasing trend, either logistically for collagen or exponentially for cells. This was combined with the outputs of a numerical model to build a function that relates gestational age to stiffness. The spatial medium generated is based on increasing the diameter and waviness of collagen fibers along with hydration and cellular content.

Hormones have been identified as the most active elements of pregnancy. The role of oxytocin, PGs, and progesterone as triggers of the whole process should always be kept in mind. More detailed monitoring of these variables can lead to improvements. The combination of cells and the proteolysis of enzyme plays a key role near childbirth. They degrade collagen while trying to maintain the integrity of an increasingly unstable structure. Thus biochemistry is able to control histology.
The viscous part of the model is irrelevant, being more sensitive to modifications in the microstructure than to the ECM content. This has shown that there is a drop in elasticity during pregnancy represented by a function with histological parameters. It can be seen that everything leads to collagen, which is why it has become the most studied component in this study. In a more general view, it is proposed to study the behavior of collagen in specimens in the absence of other constituents.

\section{Data Availability}

The data used to support the findings of this study are available from the corresponding author upon request.

\section{Conflicts of Interest}

The authors declare that there are no conflicts of interest regarding the publication of this paper.

\section{Acknowledgments}

This research was supported by the Ministry of Education [DPI2017-83859-R, DPI2014-51870-R, DPI2010-17065, and UNGR15-CE-3664], Ministry of Health [DTS15/00093 and PI16/00339, PI-0107-2017, PIN-0030-2017], Junta de Andalucía [P11-CTS-8089], and the European Social Fund (youth employment initiative) projects.

\section{References}

[1] T. Moore, E. M. Hennessy, J. Myles et al., "Neurological and developmental outcome in extremely preterm children born in England in 1995 and 2006: the EPICure studies," British Medical Journal, vol. 345, no. 7886, Article ID e7961, 2012.

[2] H. Blencowe, S. Cousens, D. Chou et al., "Born too Soon: the global epidemiology of 15 million preterm births," Reproductive Health, vol. 10, no. 1, article S2, 2013.

[3] M. S. To, Z. Alfirevic, V. C. F. Heath et al., "Cervical cerclage for prevention of preterm delivery in women with short cervix: Randomised controlled trial," The Lancet, vol. 363, no. 9424, pp. 1849-1853, 2004.

[4] C. A. Crowther, P. Ashwood, A. J. McPhee et al., "Vaginal progesterone pessaries for pregnant women with a previous preterm birth to prevent neonatal respiratory distress syndrome (the PROGRESS Study): A multicentre, randomised, placebocontrolled trial," PLoS Medicine, vol. 14, no. 9, Article ID e1002390, 2017.

[5] K. M. Myers, H. Feltovich, E. Mazza et al., "The mechanical role of the cervix in pregnancy," Journal of Biomechanics, vol. 48, no. 9, pp. 1511-1523, 2015.

[6] B. S. Oxlund, G. Ørtoft, A. Brüel et al., "Collagen concentration and biomechanical properties of samples from the lower uterine cervix in relation to age and parity in non-pregnant women," Reproductive Biology and Endocrinology, vol. 8, no. 1, p. 82, 2010.

[7] P. C. Leppert, "Anatomy and physiology of cervical ripening," Clinical Obstetrics and Gynecology, vol. 38, no. 2, pp. 267-279, 1995. 
[8] M. P. E. Wenger, L. Bozec, M. A. Horton, and P. Mesquidaz, "Mechanical properties of collagen fibrils," Biophysical Journal, vol. 93, no. 4, pp. 1255-1263, 2007.

[9] P. C. Leppert, S. Keller, J. Cerreta, Y. Hosannah, and I. Mandl, "The content of elastin in the uterine cervix," Archives of Biochemistry and Biophysics, vol. 222, no. 1, pp. 53-58, 1983.

[10] Y. Akgul, R. Holt, M. Mummert, A. Word, and M. Mahendroo, "Dynamic changes in cervical glycosaminoglycan composition during normal pregnancy and preterm birth," Endocrinology, vol. 153, no. 7, pp. 3493-3503, 2012.

[11] K. M. Myers, A. P. Paskaleva, M. House, and S. Socrate, "Mechanical and biochemical properties of human cervical tissue," Acta Biomaterialia, vol. 4, no. 1, pp. 104-116, 2008.

[12] B. Timmons, M. Akins, and M. Mahendroo, "Cervical remodeling during pregnancy and parturition," Trends in Endocrinology \& Metabolism, vol. 21, no. 6, pp. 353-361, 2010.

[13] R. Word, X. Li, M. Hnat, and K. Carrick, "Dynamics of cervical remodeling during pregnancy and parturition: mechanisms and current concepts," Seminars in Reproductive Medicine, vol. 25, no. 1, pp. 069-079, 2007.

[14] H. Feltovich, T. J. Hall, and V. Berghella, "Beyond cervical length: Emerging technologies for assessing the pregnant cervix," American Journal of Obstetrics \& Gynecology, vol. 207, no. 5, pp. 345-354, 2012.

[15] L. Peralta, G. Rus, N. Bochud, and F. S. Molina, "Mechanical assessment of cervical remodelling in pregnancy: Insight from a synthetic model," Journal of Biomechanics, vol. 48, no. 9, pp. 1557-1565, 2015.

[16] P. Pereira and L. María, "Feasibility of using ultrasonic shear waves to assess cervical remodelling during the gestation period," 2018, http://digibug.ugr.es/handle/10481/50941.

[17] M. House, D. L. Kaplan, and S. Socrate, "Relationships between mechanical properties and extracellular matrix constituents of the cervical stroma during pregnancy," Seminars in Perinatology, vol. 33, no. 5, pp. 300-307, 2009.

[18] P. Ghisletta, E. Cantoni, and N. Jacot, "Nonlinear growth curve models," in Proceedings of the International Conference on Dependent Data in Social Sciences Research Forms, Issues, and Methods of Analysis, pp. 47-66, Springer, December 2013.

[19] A. Tsoularis and J. Wallace, "Analysis of logistic growth models," Mathematical Biosciences, vol. 179, no. 1, pp. 21-55, 2002.

[20] S. Cooper, "Distinguishing between linear and exponential cell growth during the division cycle: Single-cell studies, cell-culture studies, and the object of cell-cycle research," Theoretical Biology and Medical Modelling, vol. 3, no. 1, p. 10, 2006.

[21] J. J. Moré, “The Levenberg-Marquardt algorithm: implementation and theory," in Numerical Analysis, pp. 105-116, Springer, Berlin, Germany, 1978.

[22] A. J. Fosang, C. J. Handley, V. Santer, D. A. Lowther, and G. D. Thorburn, "Pregnancy-related changes in the connective tissue of the ovine cervix," Biology of Reproduction, vol. 30, no. 5, pp. 1223-1235, 1984.

[23] E. G. Canty and K. E. Kadler, "Procollagen trafficking, processing and fibrillogenesis," Journal of Cell Science, vol. 118, no. 7, pp. 1341-1353, 2005.

[24] K. Myers, S. Socrate, D. Tzeranis, and M. House, "Changes in the biochemical constituents and morphologic appearance of the human cervical stroma during pregnancy," European Journal of Obstetrics \& Gynecology and Reproductive Biology, vol. 144, no. 1, pp. S82-S89, 2009.
[25] A. K. Williamson, A. C. Chen, K. Masuda, E. J.-M. A. Thonar, and R. L. Sah, "Tensile mechanical properties of bovine articular cartilage: Variations with growth and relationships to collagen network components," Journal of Orthopaedic Research, vol. 21, no. 5, pp. 872-880, 2003.

[26] N. S. Tavare, Industrial Crystallization: Process Simulation Analysis and Design, Springer Science \& Business Media, 2013.

[27] M. Mahendroo, "Cervical remodeling in term and preterm birth: Insights from an animal model," Reproduction, vol. 143, no. 4, pp. 429-438, 2012.

[28] M. L. Akins, K. Luby-Phelps, R. A. Bank, and M. Mahendroo, "Cervical softening during pregnancy: Regulated changes in collagen cross-linking and composition of matricellular proteins in the mouse," Biology of Reproduction, vol. 84, no. 5, pp. 1053-1062, 2011.

[29] M. C. Pinheiro, S. G. Moraes, C. N. Battlehner, E. G. Caldini, O. M. S. Toledo, and P. P. Joazeiro, "Histochemical and ultrastructural study of collagen fibers in mouse pubic symphysis during late pregnancy," Micron, vol. 35, no. 8, pp. 685-693, 2004.

[30] F. Cacho, P. J. Elbischger, J. F. Rodríguez, M. Doblaré, and G. A. Holzapfel, "A constitutive model for fibrous tissues considering collagen fiber crimp," International Journal of NonLinear Mechanics, vol. 42, no. 2, pp. 391-402, 2007.

[31] S. Jorge, S. Chang, J. J. Barzilai, P. Leppert, and J. H. Segars, "Mechanical signaling in reproductive tissues: Mechanisms and importance," Reproductive Sciences, vol. 21, no. 9, pp. 1093-1107, 2014.

[32] Y. Gan, W. Yao, K. M. Myers, J. Y. Vink, R. J. Wapner, and C. P. Hendon, "Analyzing three-dimensional ultrastructure of human cervical tissue using optical coherence tomography," Biomedical Optics Express, vol. 6, no. 4, pp. 1090-1108, 2015.

[33] G. B. de Oliveira, A. M. do Vale, A. C. dos Santos, C. E. B. de Moura, H. A. D. O. Rocha, and M. F. de Oliveira, "Composition and significance of glycosaminoglycans in the uterus and placenta of mammals," Brazilian Archives of Biology and Technology, vol. 58, no. 4, pp. 512-520, 2015.

[34] N. Uldbjerg, G. Ekman, A. Malmström, K. Olsson, and U. Ulmsten, "Ripening of the human uterine cervix related to changes in collagen, glycosaminoglycans, and collagenolytic activity," American Journal of Obstetrics \& Gynecology, vol. 147, no. 6, pp. 662-666, 1983.

[35] J. Anderson, N. Brown, M. S. Mahendroo, and J. Reese, "Utilization of different aquaporin water channels in the mouse cervix during pregnancy and parturition and in models of preterm and delayed cervical ripening," Endocrinology, vol.147, no. 1, pp.130140, 2006.

[36] J. Bercoff, M. Tanter, and M. Fink, "Supersonic shear imaging: a new technique for soft tissue elasticity mapping," IEEE Transactions on Ultrasonics, Ferroelectrics and Frequency Control, vol. 51, no. 4, pp. 396-409, 2004.

[37] L. Peralta, F. S. Molina, J. Melchor et al., “Transient elastography to assess the cervical ripening during pregnancy: a preliminary study," European Journal of Ultrasound, vol. 38, no. 4, pp. 395402, 2017.

[38] M. Muller, D. Aït-Belkacem, M. Hessabi et al., "Assessment of the cervix in pregnant women using shear wave elastography: a feasibility study," Ultrasound in Medicine \& Biology, vol. 41, no. 11, pp. 2789-2797, 2015.

[39] E. Hernandez-Andrade, A. Aurioles-Garibay, M. Garcia et al., "Effect of depth on shear-wave elastography estimated in the internal and external cervical os during pregnancy," Journal of Perinatal Medicine, vol. 42, no. 5, pp. 549-557, 2014. 
[40] L. C. Carlson, T. J. Hall, I. M. Rosado-Mendez et al., "Detection of changes in cervical softness using shear wave speed in early versus late pregnancy: An in vivo cross-sectional study," Ultrasound in Medicine \& Biology, vol. 44, no. 3, pp. 515-521, 2018.

[41] S. Y. yu, C. A. Tozzi, J. Babiarz, and P. C. Leppert, "Collagen changes in rat cervix in pregnancy-polarized light microscopic and electron microscopic studies," Proceedings of the Society for Experimental Biology and Medicine, vol. 209, no. 4, pp. 360-368, 1995.

[42] C. G. Barclay, J. E. Brennand, R. W. Kelly, and A. A. Calder, "Interleukin-8 production by the human cervix," American Journal of Obstetrics \& Gynecology, vol. 169, no. 3, pp. 625-632, 1993.

[43] O. D. Sherwood, "Relaxin's physiological roles and other diverse actions," Endocrine Reviews, vol. 25, no. 2, pp. 205-234, 2004.

[44] M. Obara, H. Hirano, M. Ogawa et al., "Changes in molecular weight of hyaluronan and hyaluronidase activity in uterine cervical mucus in cervical ripening," Acta Obstetricia et Gynecologica Scandinavica, vol. 80, no. 6, pp. 492-496, 2001.

[45] E. E. Maradny, N. Kanayama, H. Kobayashi et al., "The role of hyaluronic acid as a mediator and regulator of cervical ripening," Human Reproduction, vol. 12, no. 5, pp. 1080-1088, 1997.

[46] C. L. Elliott, J. E. Brennand, and A. A. Calder, "The effects of mifepristone on cervical ripening and labor induction in primigravidae," Obstetrics \& Gynecology, vol. 92, no. 5, pp. 804809, 1998.

[47] B. D. Byers, E. Bytautiene, M. M. Costantine et al., "Hyaluronidase modifies the biomechanical properties of the rat cervix and shortens the duration of labor independent of myometrial contractility," American Journal of Obstetrics \& Gynecology, vol. 203, no. 6, p. 596.e1, 2010.

[48] N. C. W. Hill and I. Z. Mackenzie, "2308 second trimester terminations using extra-amniotic or intra-amniotic prostaglandin E2: an analysis of efficacy and complications," BJOG: An International Journal of Obstetrics \& Gynaecology, vol. 96, no. 12, pp. 1424-1431, 1989.

[49] A. Rádestad, N. J. Christensen, and L. Strömberg, "Induced cervical ripening with mifepristone in first trimester abortion. A double-blind randomized biomechanical study," Contraception, vol. 38, no. 3, pp. 301-312, 1988.

[50] U. B. Knudsen, N. Uldbjerg, T. Rechberger, and K. Fredens, "Eosinophils in human cervical ripening," European Journal of Obstetrics \& Gynecology and Reproductive Biology, vol. 72, no. 2, pp. 165-168, 1997.

[51] G. Godeau, D. Rotten, C. Gavignet, M. C. Colin, and A. M. Robert, "Evolution of the elastic network at the cervix of the uterus during and after pregnancy," Biology of the Cell, vol. 60, no. 1, 1987.

[52] K. Myers and G. A. Ateshian, "Interstitial growth and remodeling of biological tissues: Tissue composition as state variables," Journal of the Mechanical Behavior of Biomedical Materials, vol. 29, pp. 544-556, 2014. 


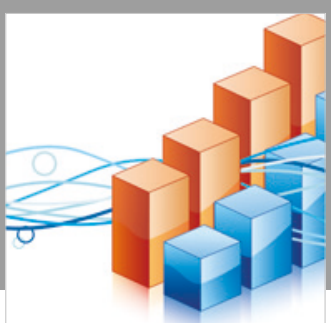

Advances in

Operations Research

\section{-n-m}
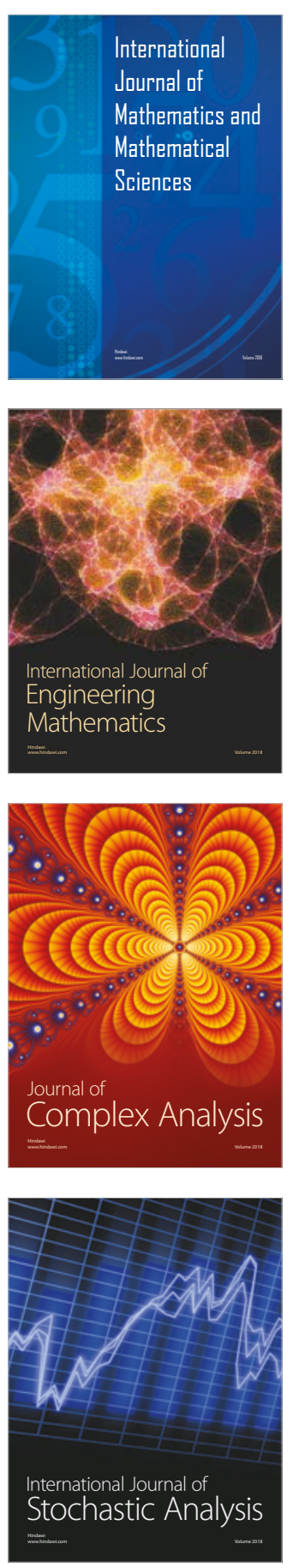
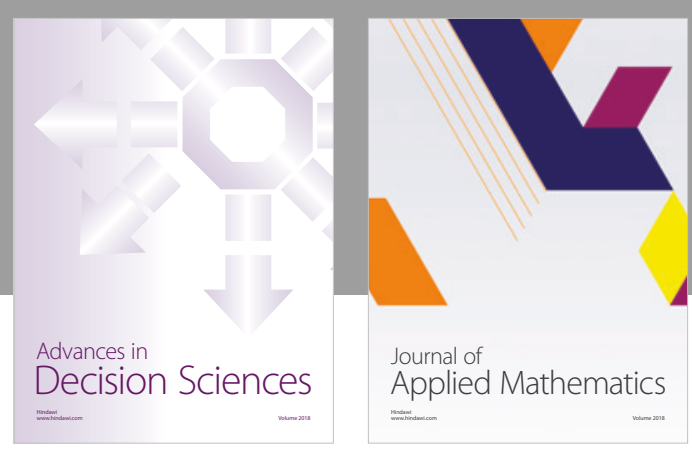

Journal of

Applied Mathematics
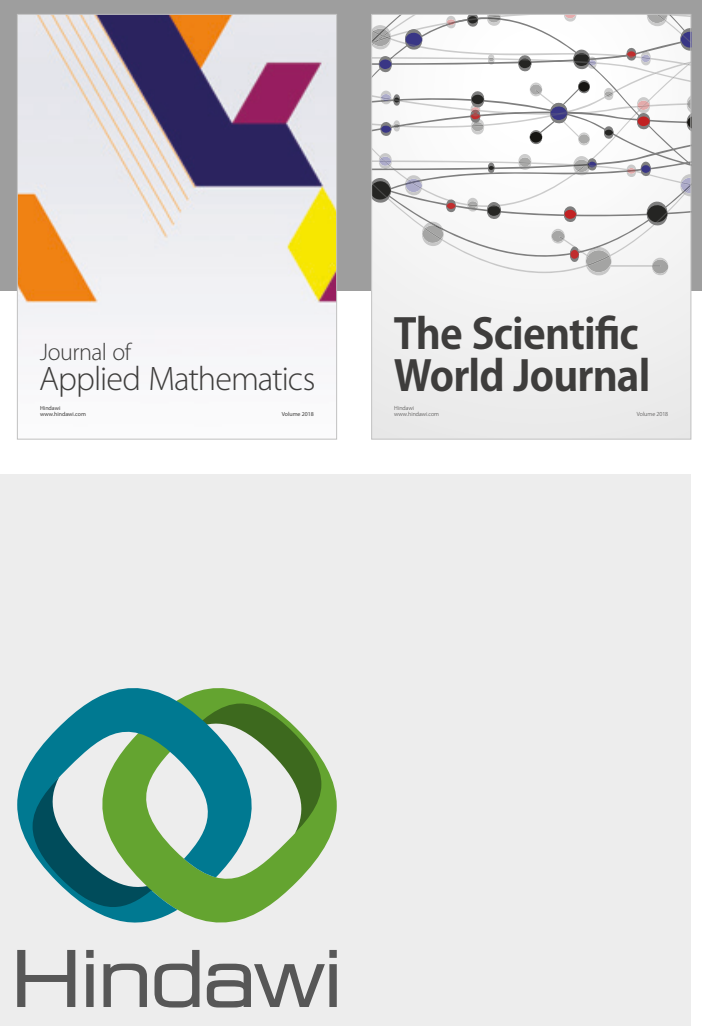

Submit your manuscripts at

www.hindawi.com

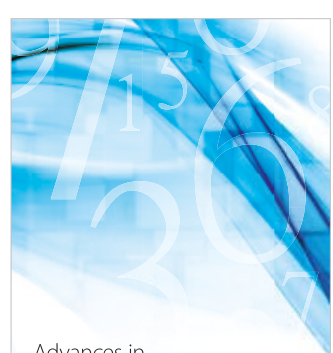

Advances in
Numerical Analysis
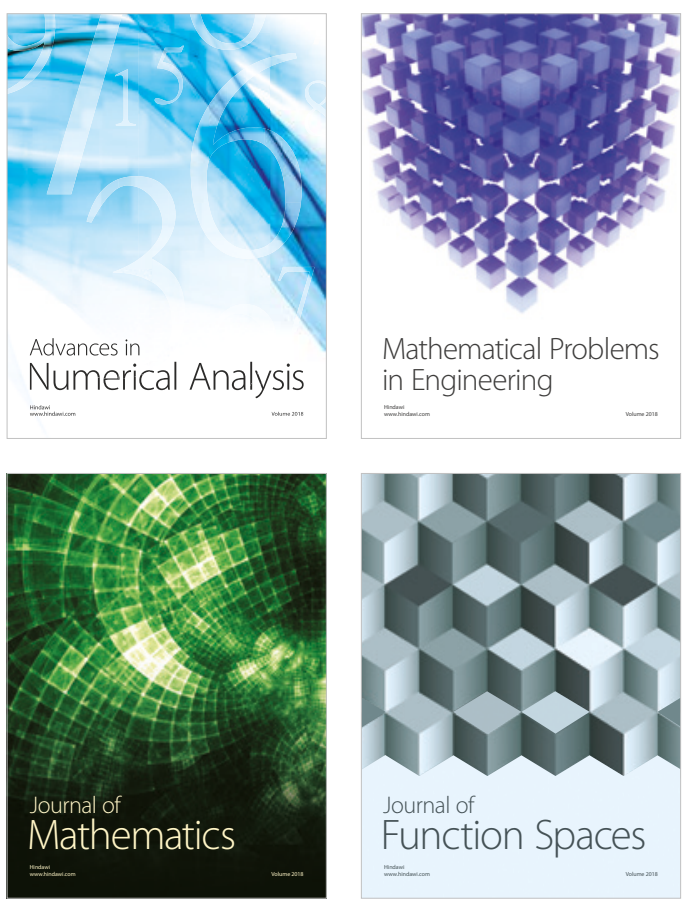

Mathematical Problems in Engineering

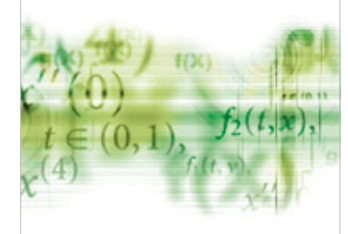

International Journal of

Differential Equations

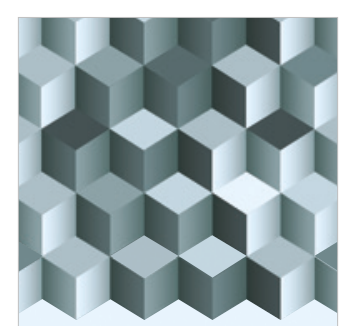

Journal of

Function Spaces

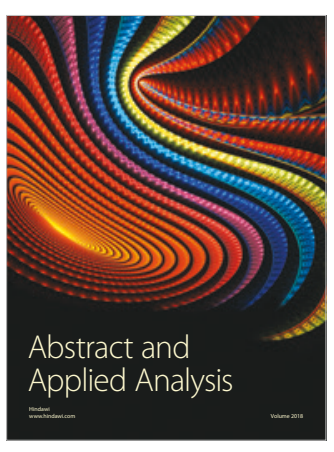

The Scientific

World Journal

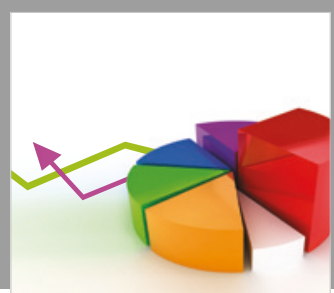

Journal of

Probability and Statistics
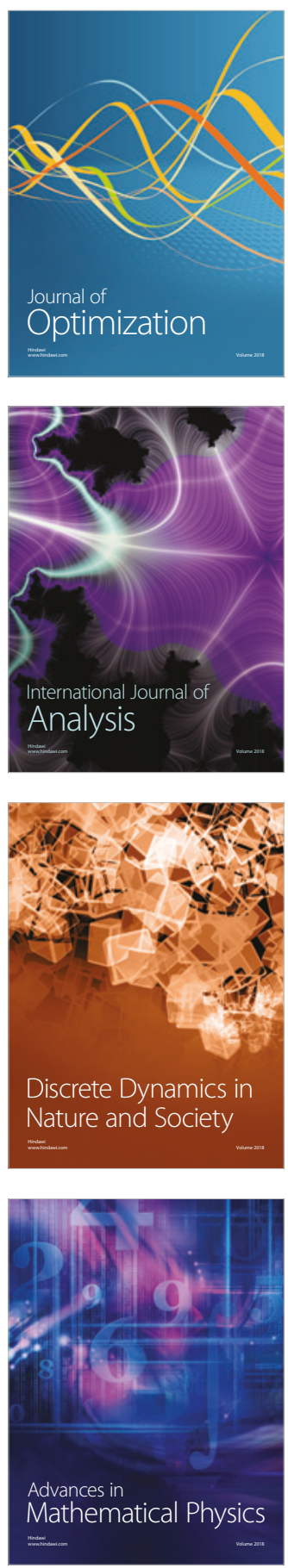